\title{
Método de control estadístico multivariante para valorar las dimensiones de calidad en una empresa de lavado de tanques industriales
}

Tomás J. Fontalvo ${ }^{*}$, Roberto Herrera ${ }^{2}$ y Enrique J. Delahoz-Dominguez ${ }^{3}$

(1) Facultad de Ciencias Económicas, Programa de Administración, Universidad de Cartagena, Campus piedra de Bolívar, Cartagena, Colombia. (correo-e: tfontalvoh@unicartagena.edu.co)

(2) Facultad de Ingenierías, Programa Ingeniería Industrial, Universidad Del Atlántico, Barranquilla, Colombia.

(correo-e: robertoherrera@mail.uniatlántico.edu.co)

(3) Facultad de Ingenierías, Programa Ingeniería Industrial, Universidad Tecnológica de Bolívar, Campus de Ternera,

Cartagena, Colombia. (correo-e: edelahoz@utb.edu.co)

* Autor a quien debe ser dirigida la correspondencia

Recibido Mar. 5, 2020; Aceptado May. 4, 2020; Versión final Jun. 24, 2020, Publicado Oct. 2020

\begin{abstract}
Resumen
Esta investigación propone un método para valorar las dimensiones de calidad del servicio en una empresa de lavado de tanques industriales, articulando una carta de control y un nuevo indicador de calidad multivariante. Como marco teórico se utilizan los conceptos de control estadístico multivariante, calidad del servicio y Seis Sigma. La información primaria corresponde a 5 dimensiones de calidad en el servicio de limpieza en 12 meses para monitorear el desempeño del servicio de forma periódica y el indicador de capacidad de manera global. La carta T2 de Hotelling evalúa las dimensiones de calidad de forma periódica y estas mostraron la mayor variabilidad en su desempeño en las dimensiones: arreglos de tanques, limpieza y pulida. En conclusión, el indicador de calidad multivariante evidenció ser pertinente para evaluar las dimensiones del servicio de forma global y precisar si el desempeño del servicio es conforme y estable.
\end{abstract}

Palabras clave: calidad del servicio; seis sigma; carta t-cuadrado

\section{Multivariate capacity indicator of quality dimensions in an industrial tank washing company}

\begin{abstract}
This research study proposes a method to assess the dimensions of service quality in an industrial tank washing company, articulating a control chart and a new multivariate quality indicator. The concepts of multivariate statistical control, service quality, and Six Sigma are used as a theoretical framework. The primary information corresponds to five quality dimensions in the cleaning service for 12 months to monitor service performance periodically and indicator capacity globally. Hotelling's T2 chart periodically assesses the quality dimensions and showed the greatest performance variability in: tank arrangements, cleaning, and polishing. In conclusion, the multivariate quality indicator proved to be adequate to globally assess service dimensions and to specify whether service performance is consistent and stable.
\end{abstract}




\section{INTRODUCCIÓN}

El transporte internacional de mercancías es una actividad vital para el desarrollo de la economía a nivel mundial, desde esta perspectiva el uso de tanques industriales estandarizados es indispensables para el transporte de todo tipo de productos, ya sea sólidos y líquidos. Consecuentemente, se ha desarrollado un sector empresarial dedicado a la limpieza de tanques estandarizados que cumplen con los estándares y medida de un contenedor. En esta investigación se evalúan las dimensiones de calidad asociadas con el servicio de limpieza de tanques de forma periódica y global, así como los criterios para identificar las variables claves que restringen el flujo normal del proceso de transporte a nivel nacional e internacional.

La importancia de valorar la calidad del servicio en una empresa de limpieza de tanques industriales radica en que, anteriormente los procedimientos de limpieza de tanques eran laboriosos, inseguros y a menudo se producían fallos en el proceso; y debido a esto han evolucionado a métodos automatizados, los cuales requieren de un análisis de calidad diferente y relacionado a su naturaleza. Para lograr objetivos económicos y cumplir con las normas de higiene, es necesario resolver problemas específicos de limpieza para lograr una solución óptima (Khalid et al, 2019). Similarmente, los autores (Holah y Saunders, 2019) coinciden en que un método de control de la calidad del servicio de limpieza permite lograr los objetivos de higiene requeridos en un marco de tiempo aceptable; lo cual evidencia la importancia del análisis de procesos de estos servicios. Los autores (Fontalvo et al, 2014) evalúan la estructura logística de las compañías que intervienen en la limpieza de contenedores y destacan la efectividad de un modelo para su objeto de estudio. Sin embargo, en el contexto no se evidencian estructuras que midan de forma periódica y de forma global el desempeño de las dimensiones de calidad, de este tipo de servicios.

De lo anterior surgen las siguientes preguntas problemas ¿Cuál serían las dimensiones de calidad que permitan evaluar el servicio de limpieza de forma periódica y de forma global? ¿Qué técnicas de control estadístico multivariante permiten evaluar de forma periódica y de forma global el desempeño de las dimensiones de calidad del servicio objeto de estudio? ¿Como articular las técnicas de control multivariante y el indicador de capacidad multidimensional para evaluar las dimensiones de calidad de forma holística? ¿Cómo contextualizar las métricas de seis sigma para evaluar el desempeño de las dimensiones de calidad del proceso de lavado de ISO tanques por medio de un indicador? ¿Como medir la variabilidad de las dimensiones de calidad de forma multidimensional de forma periódica? ¿cómo determinar y valorar un indicador de calidad multidimensional que me permita evaluar de forma integral la capacidad real de prestación de servicio de limpieza de los tanques industriales? Teniendo en cuenta lo anterior el objetivo general de esta investigación es proponer un método que articule una carta de control multivariante y un nuevo indicador de calidad multivariante en una empresa de limpieza de tanques Industriales para valorar el desempeño de las dimensiones de calidad del servicio de limpieza de forma periódica y global

Actualmente las organizaciones incorporan metodologías de medición de la calidad para mejorar su competitividad, involucrando los fundamentos de la calidad en los servicios a su estrategia organizacional. Algunos autores los relacionan por medio de normas de calidad como Capaldo et al, (2017) quienes analizan el efecto de estándares de calidad más exigentes en organizaciones proveedoras de servicios públicos, concluyendo que estas regulaciones impactan en términos de recursos y en el sistema de gestión del desempeño. Similarmente, en el estudio de Javed y llyas (2018) se integra la calidad y el servicio mediante el análisis de cinco constructos basados en las expectativas de los usuarios de una entidad pública. La importancia de la integración de la calidad y el servicio radica en que al articular las dos variables se benefician tanto a los usuarios como a los proveedores mediante el conocimiento de calificaciones del proceso. (Zhao, 2016). Por lo tanto, medir los niveles de calidad para obtener indicadores que le ayuden a la empresa a tomar decisiones objetivas basadas en datos, permite crear una ventaja por sobre sus competidores. Entonces, definir indicadores para gestionar la calidad del servicio resulta de gran impacto en las empresas. Desafortunadamente, los tomadores de decisiones en el sector de servicios no consideran como entrada las expectativas del cliente respecto al servicio prestado, por concentrarse, entre otros aspectos, como la financiación del negocio o en actividades básicas de la operación de la empresa (Causado-Rodriguez et al, 2019). El autor Hrnjic (2016) menciona que las instituciones públicas y privadas de servicios enfrentan enormes presiones económicas y escasez de capital, lo que ha llevado al buscar un balance para mantener satisfechos a sus clientes sin la necesidad de recurrir a reformas costosas.

En estudios realizados en el sector de servicios (Fazli y Muzamil, 2019), afirman que las prioridades competitivas del sector de servicios se centran en la calidad. Adicionalmente, los autores Yildiz y Kara (2015) coinciden en que, aunque las instituciones públicas no enfrentan las condiciones del mercado como empresas comerciales, para aquellas instituciones que no brindan servicios de calidad, sus esfuerzos efectivos para avanzar con los objetivos de la organización no tendrán buenos resultados. De las preguntas problemas de esta investigación surgen los siguientes objetivos específicos : i) Establecer las dimensiones de calidad para evaluar el servicio de limpieza de forma periódica y global ii) Definir las técnicas de control estadístico 
multivariante para evaluar la calidad del servicio iii) Proponer un método que articule las cartas de control multivariante y el indicador de capacidad para la calidad del servicio iv) Medir la variabilidad de las dimensiones de calidad de forma multidimensional v) Proponer y valorar un indicador de calidad multidimensional para evaluar de forma integral la capacidad real de prestación de servicio de limpieza.

\section{OTROS ANTECEDENTES}

Hay tres conceptos adicionales que es conveniente presentar y analizar para los objetivos de este trabajo: la metodología seis sigma en el servicio, el indicador de capacidad multivariante, y la carta de control multivariante T cuadrado.

\section{Seis Sigma en el servicio}

La Metodología Seis Sigma es aplicada para la mejora y evaluación de distintos sectores. Lo cual permite generar indicadores de calidad y rendimiento entre otros criterios que surgen como prerrequisito al momento de analizar contextos empresariales. Así, investigaciones previas sobre Seis Sigma se centraron en la articulación de la Manufactura esbelta y luego el papel de Lean Seis Sigma en la sostenibilidad, pero no se realiza énfasis en el campo del sector de servicios (Deshmukh et al, 2019). Sin embargo, Seis Sigma es una metodología que funciona como una herramienta robusta y objetiva, la cual lleva a la reducción de costos por medio de la prevención de defectos conduce a un aumento de la rentabilidad; proporcionando enfoques basados en evidencia que apoyan el mejoramiento de la calidad en la práctica (Davies et al, 2019).

Cuando se analizan las herramientas utilizadas por la metodología Seis Sigma, encontramos que las métricas son igualmente aplicables a la industria de los servicios. Por ejemplo en el estudio de Alkoot (2019) encontraron como el enfoque cuantitativo de Seis Sigma facilitó el diagnóstico de problemas y establece el camino para encontrar las causas y determinar mejoras. Por otro lado, en la investigación de (Zhuo 2019) la metodología Seis Sigma se aplica al sector Bancario, evidenciando como el nivel de gestión y servicio se constituyen en variables de calidad claves para medir la competitividad. Otra evidencia de su factibilidad en empresa de servicios es el estudio realizado por Arunkumar y Dillibabu (2016), quienes implementando Seis Sigma en el desarrollo de software pudieron llevar a cabo un análisis para eliminar los requisitos de los procesos sin valor e implementar la funcionalidad principal para cumplir con las expectativas del cliente.

Para Antony, et al (2019) el Seis sigma genera procesos no solo eficientes y efectivos a costos reducidos, sino también aumenta la moral de los empleados y su compromiso, ayudando a mejorar la calidad del servicio, lo cual se evidencia en resultados prometedores que contribuyen a la mejora sistemática en altos niveles. (Haerizadeh y Sunder, 2019). Cabe resaltar que, según Vashishth, et al (2017) la aplicación de Seis Sigma al servicio conlleva una serie de limitaciones que deben explorarse. Por lo tanto, es fundamental integrar Six Sigma con conceptos de calidad del servicio en la empresa de lavado de tanques industriales. Todo lo planteado anteriormente, da sentido a este trabajo de investigación que aborda un análisis de las dimensiones de calidad del servicio para una empresa de lavado de tanques Industriales

\section{Indicador de capacidad multivariante}

Chen, et al. (2003) propone el monitoreo de $v$ características, asumiendo normalidad e independencia, utilizando índice de capacidad multivariado explicado en la ecuación 1.

$$
\mathrm{CM}_{\mathrm{k}}^{\mathrm{T}}=\frac{1}{3} \emptyset^{-1}\left\{\frac{\left[\prod_{\mathrm{j}=1}^{\mathrm{v}}\left(\frac{\pi_{1}+\pi_{2}+\ldots \pi_{\mathrm{k}}}{\mathrm{k}}\right)+1\right]}{2}\right\}
$$

Esta nueva metodología presenta el porcentaje promedio de no conformes presentes en las $v$ dimensiones involucradas en el monitoreo de calidad. Este procedimiento permite evaluar el porcentaje promedio de no conformes de las $j$ th dimensiones mediante las métricas de Seis Sigma.

$$
\begin{aligned}
& \pi_{j}=\sum_{i=1}^{k} \pi_{k} / k \quad \mathrm{j}=1,2, \ldots, \mathrm{v} \\
& C \mathrm{M}_{\mathrm{k}}^{\mathrm{T}}=\frac{1}{3} \emptyset^{-1}\left\{\frac{\left[\prod_{\mathrm{j}=1}^{\mathrm{v}}\left(\frac{\left(1-\frac{N_{1}}{U_{1} \times O_{1}}\right)+\ldots+\left(1-\frac{N_{k}}{U_{k} \times O_{k}}\right)}{\mathrm{k}}\right)_{j}+1\right.}{2}\right\}
\end{aligned}
$$


Donde $\pi_{j}$ es el porcentaje promedio de no conformes en la dimensión jth y $\pi_{k}$ son las medidas de probabilidad de cada una de las categorías de la dimensión evaluada, es decir $\pi_{i}=\left(1-\left(N_{i} / U_{i} \times O_{i}\right)\right)$ con $i=1,2, \ldots, k$. Esta propuesta asume un proceso aceptable cuando el indicador es igual o mayor a 0,5 y menor que 0,75 y es excelente cuando el indicador es mayor que 0,75 , donde $\varnothing^{-1}$ es la distribución normal estándar inversa.

\section{Carta de control multivariante $T$ cuadrado}

La carta de control multivariante T-cuadrado de Hotelling (ecuación 4), ha sido analizada por diferentes autores (Herrera et al, 2019; Vargas, 2006; Vargas, 2003; Wilcox, 2001).

$$
\mathrm{T}-\text { cuadrado }=\left(\mathrm{x}_{\mathrm{i}}-\overline{\mathrm{x}}\right)^{\mathrm{t}} \mathrm{S}^{-1}\left(\mathrm{x}_{\mathrm{i}}-\overline{\mathrm{x}}\right)
$$

Donde $S$ es definida como la matriz de varianza covarianza estándar, $\bar{x}$ es el promedio de cada dimensión, $x_{i}$ es la información recolectada en el periodo. El monitoreo del rendimiento o desempeño del servicio está limitado en su fase de control, por el límite de control presentado en la ecuación 5, presentada por (Herrera, 2018; Jackson, 1985)

$$
\mathrm{CLS}=\frac{\mathrm{mv}}{(\mathrm{m}-\mathrm{v}+1)} \mathrm{F}_{\alpha,(v, \mathrm{~m}-\mathrm{v}+1)} ; \mathrm{F}_{\alpha,(v, \mathrm{~m}-\mathrm{v}+1)}
$$

En esta ecuación, $F_{\alpha,(v, m-v+1)}$ es el percentil de la distribución $\mathrm{F}$ de Fisher, $v$ es el número de dimensiones, $m$ es número de periodos, en meses. Lo anterior, permitió monitorear el desempeño del rendimiento de las dimensiones de calidad. Consecuentemente con esto, los autores (Guthrie et al, 2005) plantearon en su investigación el uso de cartas de control multivariante para medir múltiples indicadores de calidad.

\section{METODOLOGÍA}

Para el desarrollo de esta investigación se realizó una investigación de tipo valorativa, considerando que se evaluó el desempeño del servicio de limpieza de tanques industriales de forma periódica y de forma global con la carta de control y el indicador de capacidad multivariante respectivamente. Por medio de las dimensiones de calidad del proceso de limpieza de los ISO tanques. Para lo anterior se consideraron las 5 dimensiones de calidad señaladas en la Figura 1.

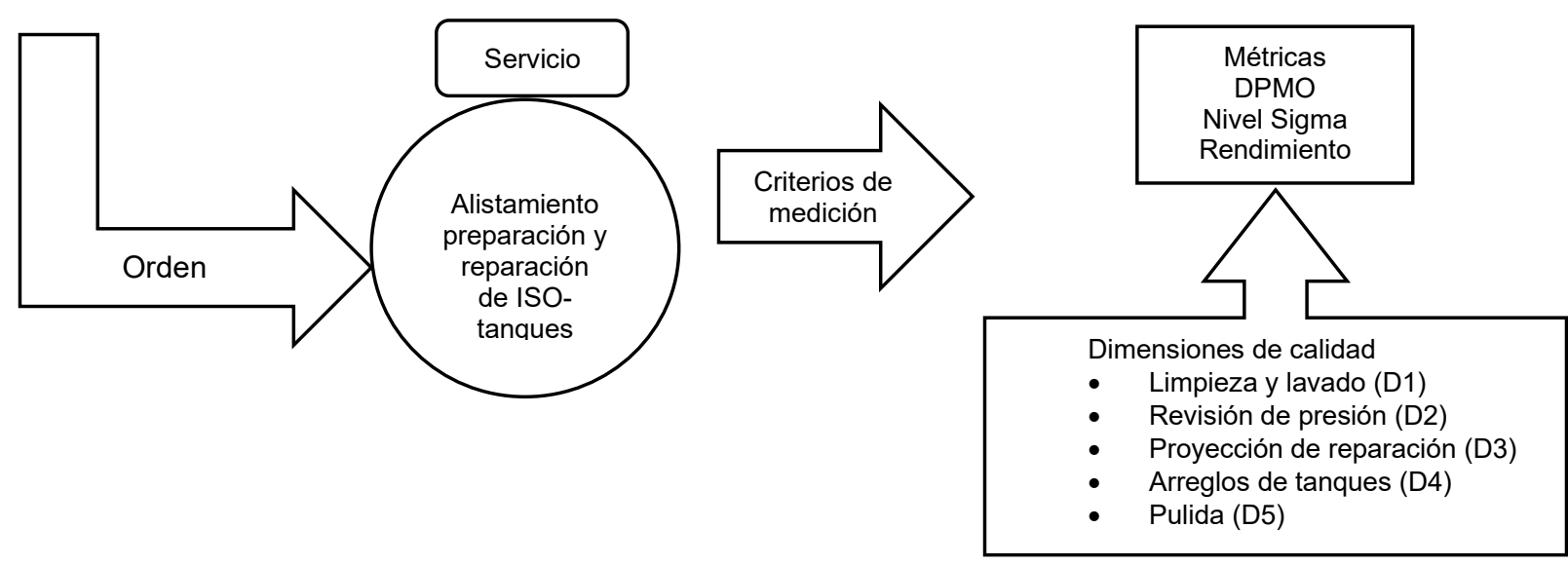

Fig. 1: Esquema de las dimensiones a evaluar las dimensiones de calidad del servicio de limpieza de los ISO tanques

Para esto se contó con información primaria del proceso, para lo anterior se tuvo la participación de los funcionarios responsables del proceso quienes aportaron la información. Seguidamente se utilizó la técnica de revisión documental y la observación para proponer el método de valoración del servicio. Para evaluar el servicio y sus dimensiones se utilizó información primaria recopilada en el proceso de limpieza de tanques de la empresa en el año 2018 (Ver Tabla 1). En la Figura 2, se presentan el método utilizado para valorar las dimensiones de calidad del servicio de forma multidimensional. Consecuentemente, se evaluaron las métricas de rendimiento para los 12 periodos del año, para así calcular el indicador multivariante. Para los cálculos se tuvo en cuenta: U: Numero de servicios prestados; O: Oportunidad de error; n: número de servicio no aceptable; Y: Rendimiento de la dimensión de calidad. 


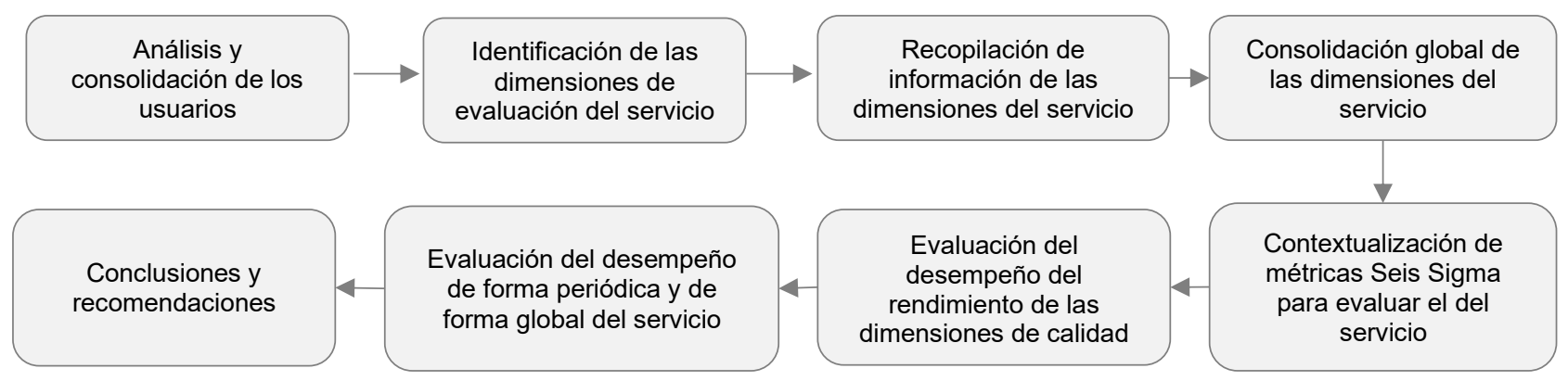

Fig. 2: Método de evaluación de las dimensiones de calidad del servicio de limpieza

En la metodología Seis Sigma, los defectos por millón de oportunidades (DPMO) se relacionan con el número real de defectos observados (ecuación 3), de esta manera se representa cuantas desviaciones estándar integran los límites de especificación del proceso del servicio, mediante la ecuación 6 . El nivel sigma se calcula mediante la ecuación 7 y el rendimiento del proceso mediante la ecuación 8.

$$
\begin{aligned}
& \text { DPMO }=\frac{\mathrm{n}}{\mathrm{t}} \times 1.000 .000=\frac{n_{i}}{U_{i} \times O_{i}} \times 1.000 .000 \\
& Z_{i}=\sqrt{ }\left(29.37-2.221 \times \ln \left(D_{P M O}\right)+0.8406\right) \\
& \pi=\left(1-\frac{n_{i}}{U_{i} \times O_{i}}\right) ; i=\text { dimensiones del servicio }
\end{aligned}
$$

\section{RESULTADOS Y DISCUSIÓN}

Para la consecución de los objetivos de esta investigación se desarrollaron dos fases, la primera requirió una propuesta y análisis de una carta de control multivariante T2 de Hotelling y la segunda fase una ii) Propuesta de un nuevo indicador de capacidad multivariante para valorar las dimensiones del servicio. Con lo que finalmente se pudo realizar un análisis periódico y global del desempeño del servicio.

Fase 1. Propuesta y análisis de una carta de control multivariante

En la Tabla 1, presentan la Información asociada con las dimensiones de Calidad del servicio analizado

\begin{tabular}{|c|c|c|c|c|c|c|}
\hline Período & Conformidad & $\mathrm{D} 1(\mathrm{O}=8)$ & $\mathrm{D} 2(\mathrm{O}=5)$ & D3 $(\mathrm{O}=4)$ & $\mathrm{D} 4(\mathrm{O}=3)$ & $\mathrm{D} 5(\mathrm{O}=6)$ \\
\hline \multirow[t]{2}{*}{ Enero } & $\mathrm{Si}$ & 277 & 277 & 277 & 270 & 19 \\
\hline & No & 21 & 10 & 9 & 20 & 1 \\
\hline \multirow[t]{2}{*}{ Febrero } & $\mathrm{Si}$ & 258 & 258 & 258 & 260 & 24 \\
\hline & No & 22 & 9 & 4 & 19 & 3 \\
\hline \multirow[t]{2}{*}{ Marzo } & $\mathrm{Si}$ & 222 & 222 & 222 & 230 & 18 \\
\hline & No & 14 & 8 & 6 & 14 & 2 \\
\hline \multirow[t]{2}{*}{ Abril } & $\mathrm{Si}$ & 247 & 247 & 247 & 240 & 22 \\
\hline & No & 16 & 7 & 6 & 14 & 2 \\
\hline \multirow[t]{2}{*}{ Mayo } & $\mathrm{Si}$ & 287 & 287 & 287 & 285 & 20 \\
\hline & No & 15 & 9 & 7 & 14 & 1 \\
\hline \multirow[t]{2}{*}{ Junio } & $\mathrm{Si}$ & 292 & 292 & 292 & 292 & 14 \\
\hline & No & 12 & 7 & 8 & 14 & 1 \\
\hline \multirow[t]{2}{*}{ Julio } & $\mathrm{Si}$ & 304 & 304 & 304 & 300 & 22 \\
\hline & No & 17 & 11 & 8 & 14 & 1 \\
\hline \multirow[t]{2}{*}{ Agosto } & $\mathrm{Si}$ & 316 & 316 & 316 & 311 & 28 \\
\hline & No & 19 & 12 & 9 & 13 & 2 \\
\hline \multirow[t]{2}{*}{ Septiembre } & $\mathrm{Si}$ & 376 & 376 & 376 & 366 & 29 \\
\hline & No & 25 & 15 & 10 & 13 & 2 \\
\hline \multirow[t]{2}{*}{ Octubre } & $\mathrm{Si}$ & 319 & 319 & 319 & 317 & 25 \\
\hline & No & 16 & 12 & 8 & 13 & 3 \\
\hline \multirow[t]{2}{*}{ Noviembre } & $\mathrm{Si}$ & 259 & 259 & 259 & 262 & 36 \\
\hline & No & 15 & 11 & 5 & 12 & 4 \\
\hline \multirow[t]{2}{*}{ Diciembre } & $\mathrm{Si}$ & 293 & 293 & 293 & 295 & 37 \\
\hline & No & 11 & 12 & 6 & 12 & 3 \\
\hline
\end{tabular}
considerando los servicios prestados a conformidad y no conformidad en los 12 meses de 20118.

Tabla 1: Información asociada con las dimensiones de Calidad del servicio analizado en la empresa de limpieza 
En la Tabla 2, los rendimientos asociados a las dimensiones de calidad durante un periodo de 12 meses. Con base en la ecuación (4) se calculó la carta de control multivariable T-cuadrado de Hotelling, para monitorear el desempeño de las variables del servicio de la empresa de limpieza de forma periódica, como se observa en la Figura 3, con lo cual se puede evaluar cada periodo de manera multidimensional, y el desempeño de la prestación del servicio, de esta manera se obtiene una visión integral y continua del comportamiento de este servicio. Es de resaltar, que en esta carta de control multivariante el contexto de las señales de alarma o los valores que están por encima del límite de control implica un aumento en la variabilidad en el desempeño de las dimensiones evaluadas integralmente en estos períodos.

Tabla 2: Cálculo del rendimiento de las dimensiones de calidad del servicio en la empresa de limpieza

\begin{tabular}{|l|l|l|l|l|l|}
\hline Mes & $\begin{array}{l}\text { Limpieza y lavado } \\
\text { (D1) }\end{array}$ & $\begin{array}{l}\text { Revisión de presión } \\
(\text { D2) }\end{array}$ & $\begin{array}{l}\text { Proyección de reparación } \\
(\text { D3) }\end{array}$ & $\begin{array}{l}\text { Arreglos de tanques } \\
\text { (D4) }\end{array}$ & $\begin{array}{l}\text { Pulida } \\
\text { (D5) }\end{array}$ \\
\hline Enero & $98,4 \%$ & $97,3 \%$ & $96,8 \%$ & $92,0 \%$ & $97,1 \%$ \\
\hline Febrero & $98,7 \%$ & $98,8 \%$ & $96,2 \%$ & $98,0 \%$ & $99,9 \%$ \\
\hline Marzo & $98,9 \%$ & $98,8 \%$ & $98,7 \%$ & $99,0 \%$ & $99,2 \%$ \\
\hline Abril & $99,1 \%$ & $94,9 \%$ & $99,1 \%$ & $97,4 \%$ & $98,7 \%$ \\
\hline Mayo & $99,0 \%$ & $99,5 \%$ & $99,1 \%$ & $97,4 \%$ & $98,9 \%$ \\
\hline Junio & $98,8 \%$ & $100,0 \%$ & $99,3 \%$ & $97,7 \%$ & $97,7 \%$ \\
\hline Julio & $97,6 \%$ & $99,5 \%$ & $99,5 \%$ & $98,3 \%$ & $96,8 \%$ \\
\hline Agosto & $96,5 \%$ & $99,5 \%$ & $98,9 \%$ & $98,6 \%$ & $93,0 \%$ \\
\hline Septiembre & $97,0 \%$ & $99,8 \%$ & $99,5 \%$ & $99,0 \%$ & $92,4 \%$ \\
\hline Octubre & $97,2 \%$ & $99,7 \%$ & $99,9 \%$ & $98,5 \%$ & $94,5 \%$ \\
\hline Noviembre & $97,5 \%$ & $99,6 \%$ & $100,0 \%$ & $99,2 \%$ & $95,1 \%$ \\
\hline
\end{tabular}

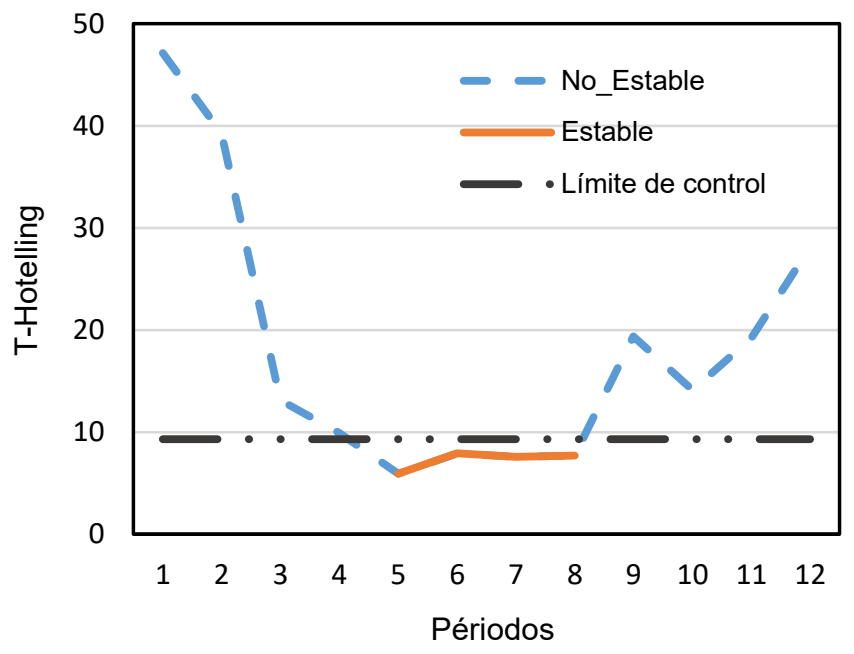

Fig. 3: Carta de control multivariante T-cuadrado de las dimensiones de calidad, no conformes

La Tabla 3, analiza la descomposición de T-cuadrado de la variabilidad total presente en el rendimiento obtenida en el servicio, la carta de control multivariante T-cuadrado de la Figura 3, evidencia que las dimensiones Arreglos de tanques y Limpieza y lavado, así como también la dimensión Pulida, presentan los valores de sumas de cuadrado más grandes, según la descomposición de T-cuadrado de la Tabla 3 , evidenciando de esta manera que son las dimensiones con mayor variabilidad en la organización en una gran parte del periodo evaluado. Con base en la ecuación (8), se calcularon los rendimientos para las diferentes dimensiones de calidad de la prestación de servicio de tanques industriales ISO TANK. Dichos resultados de rendimiento se presentan en la Tabla 4. Se puede observar que los valores paramétricos o límite de control estándar del grafico T- cuadrado está en 9,3 para los 12 periodos. Lo anterior permite definir el mínimo esperado de calidad para las dimensiones de calidad objeto de esta investigación. Esta carta de control muestra que en los meses de mayo (periodo 5), Junio (periodo 6), Julio (periodo 7), Agosto (periodo 8) los periodos presentan una variabilidad aceptable en el desempeño de estas dimensiones. Esto implica revisar la evaluación puntual de las diferentes dimensiones en estos cuatro periodos, tomar las acciones pertinentes de mejora, para que las dimensiones de los periodos 1, 2, 3,4, 9, 10,11 y 12 posean similar estabilidad. 
Tabla 3: Descomposición del estadístico T-Cuadrado

\begin{tabular}{|l|l|l|l|l|l|}
\hline $\begin{array}{l}\text { Periodo de mayor } \\
\text { variabilidad }\end{array}$ & $\begin{array}{l}\text { Limpieza y } \\
\text { lavado (D1) }\end{array}$ & $\begin{array}{l}\text { Revisión de } \\
\text { presión (D2) }\end{array}$ & $\begin{array}{l}\text { Proyección de } \\
\text { reparación (D3) }\end{array}$ & $\begin{array}{l}\text { Arreglos de } \\
\text { tanque (D4) }\end{array}$ & $\begin{array}{l}\text { Pulida } \\
\text { (D5) }\end{array}$ \\
\hline 1 & 1,132 & 2,342 & 0,455 & 36,880 & 6,510 \\
\hline 2 & 2,452 & 1,298 & 10,163 & 23,732 & 10,046 \\
\hline 3 & 0,616 & 0,656 & 6,515 & 2,281 & 9,645 \\
\hline 4 & 4,641 & 7,402 & 0,228 & 0,213 & 1,276 \\
\hline 9 & 1,108 & 1,060 & 2,869 & 7,256 & 1,670 \\
\hline 10 & 3,342 & 2,514 & 4,154 & 3,353 & 8,540 \\
\hline 11 & 3,163 & 3,537 & 0,219 & 7,703 & 6,256 \\
\hline 12 & 15,009 & 15,301 & 0,016 & 3,671 & 4,129 \\
\hline
\end{tabular}

Al revisar la Figura 3 conjuntamente a la Tabla 4, se evidencia que las dimensiones de Arreglos de tanques, Limpieza y Pulida son las que presentan mayor variabilidad. Por otro lado, cuando se analiza el elipsoide de control multivariante 3D en la Figura 4, se analiza la relación existente entre las dimensiones de mayor variabilidad en su desempeño; Arreglos de tanques y Limpieza y lavado, Pulida; este elipsoide presenta señales fuera del contorno de alarma. Lo cual requiere acciones de mejora para estas 3 dimensiones.

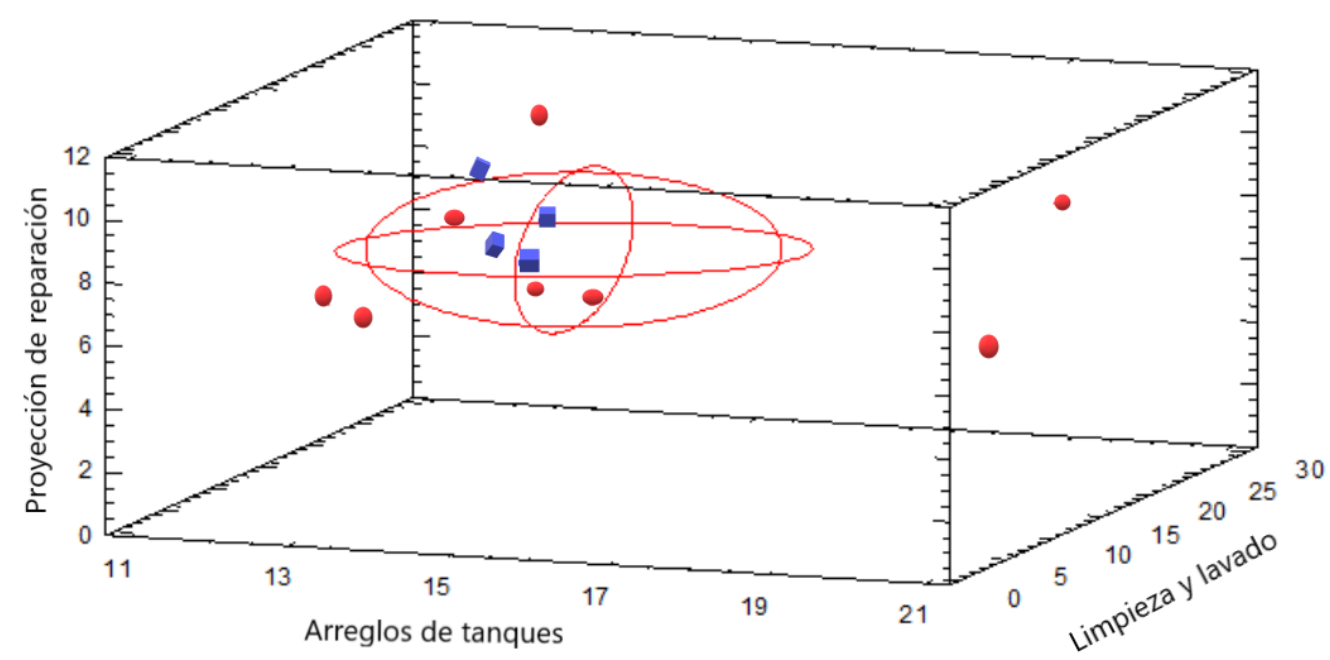

Fig. 4: Elipsoide de control multivariante 3D

De la Tabla 1 se calcularon las métricas de seis sigmas que se utilizaron para el cálculo de las métricas Seis Sigma presentadas en la Tabla 2. La etapa de valoración cuantitativa de la calidad del servicio de la empresa de limpieza se lleva a cabo mediante la apropiación de distintas ecuaciones que forman parte de la metodología matemática Seis Sigma. El resultado de las métricas de Seis Sigma mencionadas anteriormente se presenta en la Tabla 4.

Tabla 4. Métricas de Seis Sigma de las dimensiones de calidad del servicio de limpieza

\begin{tabular}{|c|c|c|c|c|c|c|c|c|c|c|c|c|c|c|c|}
\hline & \multicolumn{5}{|l|}{$D P M O$} & \multicolumn{5}{|c|}{ Nivel sigma (Z) } & \multicolumn{5}{|c|}{ Rendimiento (Y) } \\
\hline Mes & D1 & $\mathrm{D} 2$ & D3 & D4 & D5 & D1 & D2 & D3 & D4 & D5 & D1 & D2 & D3 & D4 & D5 \\
\hline Ene & 9477 & 7220 & 8123 & 12346 & 17544 & 4,9 & 5 & 4,9 & 4,9 & 4,9 & $99,1 \%$ & $99,3 \%$ & $99,2 \%$ & $98,8 \%$ & $98,2 \%$ \\
\hline Feb & 10659 & 6977 & 3876 & 12179 & 13889 & 4,9 & 5 & 5 & 4,9 & 4,9 & $98,9 \%$ & $99,3 \%$ & $99,6 \%$ & $98,8 \%$ & $98,6 \%$ \\
\hline Mar & 7883 & 7207 & 6757 & 10870 & 185 & 4,9 & 5 & 5 & 4,9 & 4,9 & $99,2 \%$ & $\%$ & 9 & $\%$ & $98,1 \%$ \\
\hline Abr & 8097 & 5668 & 6073 & 9722 & 30303 & 4,9 & 5 & 5 & 4,9 & 4,8 & $99,2 \%$ & $99,4 \%$ & $99,4 \%$ & $99,0 \%$ & $97,0 \%$ \\
\hline May & 6533 & 6272 & 6098 & 8187 & 16667 & 5 & 5 & 5 & 4,9 & 4,9 & $99,3 \%$ & $4 \%$ & $99,4 \%$ & $99,2 \%$ & $98,3 \%$ \\
\hline Jun & 5137 & 44795 & 6849 & 7991 & 23810 & 5 & 5 & 5 & 4,9 & 4,8 & $99,5 \%$ & $99,5 \%$ & $99,3 \%$ & $99,2 \%$ & $97,6 \%$ \\
\hline Jul & 6990 & 7237 & 6579 & 7778 & 22727 & 5 & 5 & 5 & 4,9 & 4,8 & $99,3 \%$ & $3 \%$ & $99,3 \%$ & $99,2 \%$ & $97,7 \%$ \\
\hline Ago & 7516 & 7595 & 7120 & 6967 & 23810 & 4,9 & 4,9 & 5 & 5 & 4,8 & $99,2 \%$ & $99,2 \%$ & $99,3 \%$ & $99,3 \%$ & $97,6 \%$ \\
\hline Sep & 8311 & \begin{tabular}{|l|}
7979 \\
\end{tabular} & 6649 & 5920 & 22989 & 4,9 & 4,9 & 5 & 5 & 4,8 & $99,2 \%$ & $99,2 \%$ & $99,3 \%$ & $99,4 \%$ & $97,7 \%$ \\
\hline Oct & 6270 & \begin{tabular}{|l|}
7524 \\
\end{tabular} & 6270 & 6835 & 40000 & 5 & 5 & 5 & 5 & 4,8 & $99,4 \%$ & $99,2 \%$ & $99,4 \%$ & $99,3 \%$ & $96,0 \%$ \\
\hline Nov & 7239 & 8494 & 4826 & 7634 & 37037 & 5 & 4,9 & 5 & 5 & 4,8 & $99,3 \%$ & $99,2 \%$ & $99,5 \%$ & $99,2 \%$ & $96,3 \%$ \\
\hline Dic & 4693 & 8191 & 5119 & 6780 & 27027 & 5 & 4,9 & 5 & 5 & 4,8 & $99,5 \%$ & $99,2 \%$ & $99,5 \%$ & $99,3 \%$ & $97,3 \%$ \\
\hline
\end{tabular}




\section{Fase 2. Propuesta de un nuevo indicador de capacidad multivariante}

A continuación, se presentan los cálculos para valorar las dimensiones de calidad del servicio de limpieza por medio del nuevo indicador de capacidad multivariante propuesto. En las Tablas 4 se observan el cálculo de las métricas de Seis Sigma en todas las dimensiones de calidad del servicio de limpieza de tanque para los doce meses de 2018. Los cuales funcionan como insumos para el cálculo del nuevo indicador propuesto objeto de estudio en este ítem.

\section{Análisis de indicador de capacidad multidimensional}

La nueva propuesta de indicador de capacidad multidimensional, propuesto en esta investigación, según las ecuaciones (1), (2) y (3) permiten obtener los resultados del indicador de capacidad multivariable. El resultado se presenta en las ecuaciones (9) y (10)

$$
\begin{aligned}
\mathrm{CM}_{\mathrm{k}}^{\mathrm{T}}= & \frac{1}{3} \emptyset^{-1}\left\{\frac{\left[\prod_{\mathrm{j}=1}^{\mathrm{v}}\left(\frac{0.990523+. .+0.982456}{5}\right) \times \ldots \times\left(\frac{0.995307+. .+0.972973}{5}\right)_{j}+1\right]}{2}\right\} \\
\mathrm{CM}_{\mathrm{k}}^{\mathrm{T}} & =\frac{1}{3} \emptyset^{-1}\left\{\frac{[(0,989058 \times \ldots \times 0.989638)+1]}{2}\right\}==\frac{1}{3} \emptyset^{-1}\left\{\frac{(0.8782)+1]}{2}\right\}=0.516
\end{aligned}
$$

La valoración del indicador de capacidad de calidad multivariante obtuvo una calificación de 0.516 muestra que la prestación del servicio evaluada integralmente muestra un desempeño bueno, de acuerdo con los criterios previamente definidos. Sin embargo, es necesario tomar acciones de mejora, específicamente en las dimensiones de Arreglos de tanques y Limpieza y lavado, Pulida en los periodos. La evidencia empírica asociada con las cartas de control multivariante utilizada permite señalar la pertinencia para monitorear el desempeño periódico de múltiples dimensiones de calidad del servicio analizado. De igual manera permite señalar que el desempeño global del servicio prestado analizado en los 12 periodo es bueno toda vez que el indicador de capacidad multivariante obtuvo un valor superior a 0,5 . Lo que permite señalar la pertinencia, coherencia e integralidad de utilizar las dos técnicas de control estadístico multivariante para valorar el servicio de limpieza de tanques industriales, desde dos perspectivas complementarias.

Por otro lado, los resultados obtenidos en la presente investigación son coherentes con otras investigaciones en contextos similares. Por ejemplo, Shahriari y Abdollahzadeh (2009) por medio de un vector de capacidad de proceso multivariante evaluaron la precisión y rendimiento del proceso, concluyendo que cuando las características están altamente correlacionadas los resultados globales mejoran. En la investigación de Liu et al. (2019) se identificaron variaciones temporales y espaciales en parámetros complejos de calidad y en diferentes cargas de factores, convirtiendo enormes estructuras de parámetros en estructuras de factores más simples. Similarmente, este tipo de indicador resultó practico para monitorear la estabilidad del proceso, así como menciona Herrera et al, (2018) quien por medio de esta herramienta evaluó el cumplimiento de los parámetros de un proceso metalúrgico, midiendo la variabilidad del proceso durante diferentes fases.

En el caso de (Delahoz-Dominguez et al, 2020), en su implementación del proceso Seis Sigma en una universidad, evalúan cinco dimensiones de calidad, encontrando un desempeño promedio del proceso del $93.27 \%$, resultado inferior al de la presente investigación. En contraste con estos resultados, otros autores han utilizado otras perspectivas de calidad para generar mejoras de calidad en empresas de servicios. (Fontalvo y De la Hoz, 2018) desde análisis racionales. Es importante desatacar de esta investigación que a diferencia de la presentada por los investigadores (Fontalvo et al, Prensa) en donde solo se hace un estudio desde una perspectiva de desempeño puntual. En esta investigación, se aborda el análisis de las dimensiones del servicio desde dos perspectivas, se realiza un análisis longitudinal y otro específico y global, lo cual hace al método más pertinente para valorar el servicio.

\section{CONCLUSIONES}

1.- La evidencia empírica permite valorar el desempeño del servicio de limpieza en la empresa como bueno. Considerando el resultado del indicador de capacidad multivariante mayor a 0,5 , el cual evaluó las cinco dimensiones de calidad del servicio en los 12 meses de la investigación. Sin embargo, se pudo identificar por medio de la carta de control La carta T2 de Hotelling, que en los meses 1,2,3,4, 9,10,11, las dimensiones de calidad del servicio presentan variabilidad. 
2.-El modelo propuesto es pertinente para monitorear de forma periódica y de forma global las dimensiones de calidad de la empresa de limpieza objeto de estudio, esto es debido a la complementariedad de las dos técnicas integradas para valorar las dimensiones del servicio objeto de esta investigación de manera holística.

3.-Como contribución única de esta investigación se aporta un método, el cual integra dos fases y dos técnicas de control estadístico multivariante, es novedoso, toda vez que es pertinente para organizaciones de servicios y no solo como tradicionalmente se utilizan estas cartas de control en empresas de producción.

4.- Como valor agregado y aporte científico de esta investigación se aporta un método estructurado que integra una carta de control multivariante y un nuevo indicador de capacidad multidimensional para evaluar las dimensiones de calidad de un servicio de limpieza de forma periódica y de forma global; en términos de conocimiento es un aporte significativo al articular conceptos de cartas de control e indicadores de capacidad multidimensionales, asociadas con el control estadístico en el sector de servicios.

5.- Por otro lado, el indicador propuesto es positivamente conservador, es decir, se debe tener en el proceso rendimientos altos, para obtener indicadores superiores a 0.5 , por tanto, es de mucha utilidad, cuando se necesite mejorar la calidad de los procesos, especialmente cuando se evalúa prestación de servicios.

\section{REFERENCIAS}

Alkoot, F.M., Using 6 sigma to improve outcomes of higher education institutes. https://doi.org/10.18178/ijiet.2019.9.1.1171, International Journal of Information and Education Technology, 9(1), 46-50 (2019)

Antony, J., Rodgers, B. y Cudney, E., Lean Six Sigma in policing services: case examples, lessons learnt and directions for future research. https://doi.org/10.1080/14783363.2017.1327319,Total quality management \& business excellence, 30(5-6), 613-625 (2019)

ArunKumar, G. y Dillibabu, R., Design and Application of New Quality Improvement Model: Kano Lean Six Sigma for Software Maintenance, https://doi.org/10.1007/s13369-015-1933-1, Project Arab J Sci Eng, 41, 997-1014 (2016)

Capaldo, G., Costantino, N. y Pellegrino, R., The Effect of More Demanding Public Services Quality Standards on the Organization of Service Providers. https://doi.org/10.1080/01900692.2017.1295263, International Journal of Public Administration, 40(10), 847-859 (2017)

Causado-Rodriguez, E., Charris, A.N. y Guerrero, E.A., Mejora Continua del Servicio al Cliente Mediante ServQual y Red de Petri en un Restaurante de Santa Marta, Colombia. https://doi.org/10.4067/S0718-07642019000200073, Información tecnológica, 30(2), 73-84 (2019)

Davies, C., Lyons, C. y Whyte, R., Optimizing nursing time in a day care unit: Quality improvement using Lean Six Sigma methodology. https://doi.org/10.1093/intqhc/mzz087, International journal for quality in health care : journal of the International Society for Quality in Health Care, 31(1), 22-28 (2019)

De la hoz-Dominguez, E., Fontalvo, T. y Fontalvo, O., Evaluación de la calidad del servicio por medio de seis sigma en un centro de atención documental en una Universidad. https://doi.org/10.4067/S0718-50062020000200093, Formación Universitaria, 13(2), 93-102 (2020)

Deshmukh, V.K., Mukti, S.K. y Agrawal, A., Applicability of Lean Six Sigma in Hospitals, Advances in Industrial and Production Engineering, Lecture Notes in Mechanical Engineering, 861-870 (2019)

Fazli, I. y Muzamil, M., Exploring competitive priorities in the service sector: evidence from India. https://doi.org/10.1108/IJQSS-02-2018-0021, International Journal of Quality and Service Sciences, 11(2), 167-186 (2019)

Fontalvo, T.J., Cardona, D. y Morelos, J., Aplicación del modelo scor para el servicio de limpieza de contenedores tanque (iso tanks), Revista Dimensión Empresarial, 12(2), 59-68 (2014)

Fontalvo, T.J. y De la Hoz, E., Diseño e Implementación de un Sistema de Gestión de la Calidad ISO $9001: 2015$ en una Universidad Colombiana. https://doi.org/10.4067/S0718-50062018000100035, Formación universitaria, 11(1), 35-44 (2018)

Fontalvo, T., Herrera, R., y De la Hoz, E., Método de evaluación de la calidad del servicio de una unidad de atención al usuario en una empresa de servicio de agua en Colombia, Información Tecnológica, 31(4) (Prensa)

Guthrie, G., Love, T. y otros 3 autores, Compare and communicate: designing control charts to summarise efficiently data from multiple quality indicators, Qual Saf Health Care,14 (6), 450-454 (2005)

Haerizadeh, M. y Sunder, V., Impacts of Lean Six Sigma on improving a higher education system: a case study. https://doi.org/10.1108/IJQRM-07-2018-0198, International Journal of Quality \& Reliability Management, 36(6), 983-998 (2019)

Herrera, R.J., Rojas, P.M. y Jiménez, K.P., Cartas de control con variables convolucionadas, I+D Revista de Investigaciones, 13 (1), 82-87 (2019) 
Herrera-Acosta, R., Hernández-Alvear, K., Figueroa-Gélvez, E. y De La Ossa-De Ávila, J., Application of multivariate statistical control to measure the process capability of compression springs in stainless steel, Prospectiva, 16(2), 49-58, 2018

Holah, J. y Saunders, D., Open Plant Cleaning Methods \& Equipment. https://doi.org/10.1016/B978-0-08-100596-5.212055, Reference Module in Food Science, (2019)

Hrnjic, A., The transformation of higher education: evaluation of CRM concept application and its impact on student satisfaction. https://doi.org/10.1007/s40821-015-0037-x, Eurasian Bus Rev, 6, 53-77 (2016)

Jackson, J. E., Multivariate Quality Control, Communications in Statistics, 14, 2657-2688 (1985)

Javed, S. y llyas, F., Service quality and satisfaction in healthcare sector of Pakistan - the patients' expectations. https://doi.org/10.1108/IJHCQA-08-2016-0110, International Journal of Health Care Quality Assurance, 31(6), 489-501 (2018)

Khalid, N.I., Saulaiman, U.S. y otros 8 autores, Integrating cleaning studies with industrial practice: Case study of an effective cleaning program for a frozen meat patties SME factory, https://doi.org/10.1016/j.jclepro.2019.06.317, Journal of Cleaner Production, 235, 688-700 (2019)

Liu, Z., Chul, J. y otros 3 autores, Assessment of water quality and Algae growth for the Ganwol reservoir using multivariate statistical analysis, https://doi.org/10.1080/15715124.2019.1672703, International Journal of River Basin Management, (2019)

Shahriari, H. y Abdollahzadeh, M., A new multivariate process capability vector, Quality Engineering, 21, 290-299 (2009)

Vargas, J.A., Control Estadístico de Calidad, Universidad Nacional de Colombia, Bogotá, (2006)

Vargas, J.A., Robust estimation in multivariate control charts for individual observations. https://doi.org/ 10.1080/00224065.2003.11980234, J. Quality Technology, 35(4), 367-376 (2003)

Vashishth, A., Chakraborty, A. y J. Antony, Lean Six Sigma in financial services industry: a systematic review and agenda for future research, Total Quality Management \& Business Excellence, 30(3-4), (2017)

Wilcox, R., Fundamentals of modern statistical methods, Springer, New YorkCross (2001)

Yildiz, S.M. y Kara, A., Developing Alternative Measures for Service Quality in Higher Education: Empirical Evidence from the School of Physical Education and Sports Sciences, Proceedings of the 2009 Academy of Marketing Science (AMS) Annual Conference, 185-185 (2015)

Zhao, G., Qian, X., Lei, X. y T. Mei, Service Quality Evaluation by Exploring Social Users' Contextual Information. https://doi.org/10.1109/TKDE.2016.2607172, IEEE Transactions on Knowledge and Data Engineering, 28(12), 3382-3394 (2016)

Zhuo, Z., Research on using Six Sigma management to improve bank customer satisfaction, https://doi.org/10.1186/s40887-019-0028-6, International Journal of Quality Innovation, 5(3), (2019) 